\title{
Analysis of Clinical Effects of Huangqi Maidong Decoction Combined with Insulin Aspart Injection in the Treatment of Gestational Diabetes
}

Zhuo Liu*

Guangxi University of Traditional Chinese Medicine, Nanning 530011, China. E-mail: daphne0825@163.com

\begin{abstract}
Objective: This article mainly explores the therapeutic effect of Huangqi Maidong Decoction combined with aspart insulin injection on patients with gestational diabetes. Methods: The study period was from January 20, 2017 to December 15, 2018. A total of 156 patients with gestational diabetes mellitus admitted in this hospital were selected and grouped by the randomization principle. They were grouped by randomization into the Chinese medicine group ( $\mathrm{n}=52$ cases), the western medicine group ( $n=52$ cases) and the study group ( $n=52$ cases), and patients in the Chinese medicine group were treated with Huangqi Maidong Decoction. Patients in the western medicine group were treated with aspart insulin injection. And patients in the study group were treated with Huangqi Maidong Decoction combined with aspart insulin injection to help patients to recover. Four weeks are taken as a course of treatment. After one course of treatment, the three groups of patients were compared for blood glucose control and delivery mode. It is impossible to predict the outcome of pregnancy and the corresponding results. After one course of treatment, the blood glucose control, delivery mode and pregnancy outcome of the study group was better than those of the traditional Chinese medicine group and western medicine group, which was statistically significant $(\mathrm{P}<0.05)$. And asphyxia occurred in the study group after the intervention. Pneumonia and hypoglycemia were significantly less than those in the traditional Chinese medicine group and the western medicine group with statistical significance $(\mathrm{P}<0.05)$. Conclusion: In the clinical treatment of gestational diabetic patients, the clinical effect of Huangqi Maidong Decoction combined with aspart insulin injection is obvious. It can control the blood sugar of the patient, promote the natural delivery of the mother, and improve the pregnancy outcome and newborn outcome to ensure the safety of mothers and infants.
\end{abstract}

Keywords: Huangqi Maidong Decoction; Insulin Aspart Injection; Gestational Diabetes; Clinical Effect

\section{Conclusion}

Gestational diabetes, gestational diabetes mellitus (GDM) is a kind of very common current clinical pregnancy complications, and it is a type of diabetes. ${ }^{[1]}$ If not treated and controlled, fetal deformity and death will increase significantly, newborns may also suffer from complications such as hypoglycemia and macrosomia, and may even lead to maternal dystocia or death. ${ }^{[2,3]}$
Some studies have shown that the probability of gestational diabetes in China's pregnant women is about $1 \%-5 \%$. With the continuous improvement of people's living standard in recent years, the incidence of GDM has been on the rise year by year. ${ }^{[4,5]}$ At present, the clinical treatment for gestational diabetes is mainly to adjust the diet and exercise of pregnant women, or to follow the doctor's advice to take oral hypoglycemic drugs, such as metformin. However, as the pathogenesis of the disease

\footnotetext{
Copyright (C) 2020 Zhuo Liu

doi: $10.18686 /$ aem.v9i1.159

This is an open-access article distributed under the terms of the Creative Commons Attribution Non-Commercial License (http://creativecommons.org/licenses/by-nc/4.0/), which permits unrestricted non-commercial use, distribution, and reproduction in any medium, provided the original work is properly cited.
} 
is complex and the onset period is relatively special, the simple use of western medicine to control blood sugar is not ideal. ${ }^{[6]}$ In recent years, with the development of Chinese medicine, the application of TCM in the treatment of GDM has attracted more and more attention. ${ }^{[7]}$ Gestational diabetes belongs to the category of "relieving thirst during pregnancy" in traditional Chinese medicine. The main cause of the disease is that due to the diet of pregnant women and the accumulation of energy and blood to nourish the fetus, and the deficiency of maternal energy and Yin causes the disease. Astragalus ophiopogonis decoction is a common prescription of nourishing Yin and energy in traditional Chinese medicine. ${ }^{[8]}$ In this study, 156 pregnant women with gestational diabetes admitted to our hospital from January 20, 2017 to December 15, 2018 were randomly divided into TCM group ( $\mathrm{n}=52$ cases), western group ( $n=52$ cases) and research group ( $n=52$ cases). The pregnant women in the Chinese medicine group were treated with astragalus ophiopogonis decoction, and the pregnant women in the western medicine group were treated with Mendong insulin injection. On this basis, the pregnant women in the research group were treated with astragalus ophiopogonis decoction and Mendong insulin injection to help them control their blood sugar. Four weeks is a course of treatment. After a course of treatment, observing the nutritional status and various indicators of the three groups of pregnant women, in order to explore the effect of Huangqi Maidong decoction and Mendong insulin injection on the treatment of pregnant women with gestational diabetes, and the details are reported below.

\section{Materials and methods}

\subsection{General information}

The study was conducted from January 20, 2017 to December 15, 2018. 156 pregnant women with gestational diabetes admitted to our hospital were randomly divided into Chinese medicine group $(n=52$ cases), western medicine group ( $\mathrm{n}=52$ cases $)$ and research group ( $\mathrm{n}=52$ cases).

In the TCM group, 52 patients were aged 24-38 years, with an average age of $29.79 \pm 2.48$ years. The average weight was $(65.25 \pm 9.66) \mathrm{kg}$. Gestational weeks were 24-34 weeks, with an average of $29.37 \pm 1.33$ weeks.
In the western medicine group, 52 patients were aged 20-38 years, with an average age of $25.79 \pm 2.32$ years. The average weight was $(60.25 \pm 8.56) \mathrm{kg}$. Gestational age was 25-35 weeks, with an average of $29.53 \pm 1.32$ weeks.

Fifty-two patients in the study group were aged 23-39 years with an average age of $(30.12 \pm 3.01)$ years. The body weight was $44-87 \mathrm{~kg}$, and the average weight was $(65.67 \pm 9.52) \mathrm{kg}$. Gestational weeks ranged from 24 to 34 weeks with an average of $29.19 \pm 1.28$ weeks.

In order to ensure the rigor of the data in this study, the consent of pregnant women had been obtained before the survey. The comparative analysis of relevant data and the data of pregnant women themselves showed that there was no significant difference in maternal conditions among the three groups $(\mathrm{P}>0.05)$.

\subsection{Inclusion and exclusion criteria}

Inclusion criteria: (1) conform to GDM diagnostic criteria; ${ }^{[9]}$ (2) sign the "informed consent"; (3) normal and conscious.

Exclusion criteria: (1) the presence of continued pregnancy contraindications; (2) abnormal liver and kidney function; (3) the existence of liver dysfunction or abnormal coagulation mechanism; (4) patients who have been diagnosed with diabetes before pregnancy.

\subsection{Methods}

In this study, 156 pregnant women were treated with conventional methods, which required them to make a reasonable diet plan for pregnant women according to their blood glucose status, to eat little and often, and to maintain moderate exercise every day.

Chinese medicine group: 52 pregnant women were treated with astragalus ophiopogonis decoction, the prescription was: Astragalus 30g, Ophiopogonis 20g, Chinese Yam 20g, Ejiao 18g, Poria Cocos 20g, Cooked Rehmannia Rehmandii 20g, Schisandrae 12g, Huangjing 10g, Yuzhu 12g. ${ }^{[10]}$ Method of administration: choose non-decoction traditional Chinese medicine (produced by Jiangsu Jiangyin Tianjiang pharmaceutical co., LTD.), one set per day, rinse with water to $200 \mathrm{ml}$, and take it twice in the morning and evening, $100 \mathrm{ml}$ each time.

Western medicine group: 52 pregnant women were treated by subcutaneous injection of Mendong insulin in the islet group. Mendong insulin was selected for 
subcutaneous injection of $0.3-0.8 \mathrm{U} / \mathrm{kg}$ in pregnant women. Insulin was injected twice a day, one third of the total insulin needed for the day before breakfast and one third of the total insulin needed for the day before dinner. The final step is to adjust insulin dosage according to blood glucose during pregnancy.

Study group: 52 pregnant women were treated with astragalus ophiopogonis decoction and Mendong insulin injection.

The three groups of pregnant women took 4 weeks as a course of treatment, after a course of treatment, the pregnant women's indicators were evaluated in order to observe the application effect of nutrition assessment and special disease integrated diet guidance.

\subsection{Evaluation index}

After a course of treatment, blood glucose control, pregnancy outcome and neonatal outcome were compared among the three groups.

\subsection{Statistical analysis}

SPSS24.0 software was used to expand data processing and measurement data. The expression form was: $t$ test. The enumeration data, expressed in the form of $[\mathrm{n}(\%)]$, were mainly tested, $\mathrm{P}<0.05$, showing a statistical difference $x^{2}$.

\section{Results}

\subsection{Comparison of blood glucose control after a course of treatment}

After a course of treatment, the blood glucose control of pregnant women in the study group was better than that of the Chinese medicine group and the western medicine group, showing statistical significance $(\mathrm{P}<$ 0.05). The specific scoring results are shown in Table 1.

\begin{tabular}{|c|c|c|c|c|c|}
\hline \multirow[t]{2}{*}{ Group } & \multirow{2}{*}{$\begin{array}{l}\text { The number of } \\
\text { cases }\end{array}$} & \multicolumn{2}{|l|}{ FPG } & \multicolumn{2}{|l|}{2 HPG } \\
\hline & & Baseline & $\begin{array}{l}\text { After a period of } \\
\text { treatment }\end{array}$ & Baseline & $\begin{array}{ll}\text { After a period of } \\
\text { treatment }\end{array}$ \\
\hline Traditional & $\mathrm{N}=52$ & $7.28+1-$ & $4.78+/-1.01^{\mathrm{ab}}$ & $11.37+/-1.45$ & $6.98+/-0.84^{\mathrm{ab}}$ \\
\hline Chinese & & 1.11 & & & \\
\hline \multicolumn{6}{|l|}{ Medicine } \\
\hline \multicolumn{6}{|l|}{ Group } \\
\hline Western & $\mathrm{N}=52$ & $7.26+1-$ & $4.98+/-1.04^{\mathrm{ab}}$ & $11.42+/-1.49$ & $7.57+/-0.85^{\mathrm{ab}}$ \\
\hline Medicine & & 1.09 & & & \\
\hline \multicolumn{6}{|l|}{ Group } \\
\hline \multirow[t]{2}{*}{ Study Group } & $\mathrm{N}=52$ & $7.31+1-$ & $4.19+/-0.95^{\mathrm{a}}$ & $11.35+/-1.43$ & $6.36+/-0.64^{\mathrm{a}}$ \\
\hline & & 1.12 & & & \\
\hline
\end{tabular}

Table 1. Blood glucose control in the three groups $(\mathrm{x} \pm \mathrm{s})$

Note: Compared with baseline, $P<0.01$; $^{a}$ Compared with the study group, $P<0.01 .^{b}$

\subsection{Comparison of pregnancy outcomes among the three groups after a course of treatment}

After a period of treatment, comparing the pregnancy outcomes of pregnant women in the three groups, it can be found that the pregnancy outcomes of pregnant women in the study group were significantly better than those in the traditional Chinese medicine group and the western medicine group, with statistical significance $(\mathrm{P}<0.05)$. The specific results are shown in Table 2. 


\begin{tabular}{llllll}
\hline Group & $\begin{array}{l}\text { The number of } \\
\text { cases }\end{array}$ & Preeclampsia & Hydramnios & Macrosomia & Preterm Birth \\
\hline $\begin{array}{l}\text { Traditional } \\
\text { Chinese Medicine }\end{array}$ & $\mathrm{N}=52$ & $6(11.54)$ & $8(15.38)$ & $6(11.54)$ & $3(5.77)$ \\
Group & & & & & $2(3.85)$ \\
Western Medicine & $\mathrm{N}=52$ & $5(9.62)$ & $9(17.31)$ & $8(15.38)$ & $0(\mathrm{zero})$ \\
Group & $\mathrm{N}=52$ & $3(5.77)$ & $4(7.69)$ & $5(9.62)$ & 8.78 \\
Study Group & & 14.05 & 12.96 & 11.49 & $<0.05$ \\
$\mathrm{X}^{2}$ & & $<0.05$ & $<0.05$ & $<0.05$ & \\
$\mathrm{P}$ & & & & & \\
\hline
\end{tabular}

Table 2. Comparison of pregnancy outcomes among three groups [n (\%)]

\subsection{Comparison of neonatal outcomes after a course of treatment}

After a course of treatment, the neonatal outcome of the study group was better than that of the Chinese medicine group and the western medicine group, showing statistical significance $(\mathrm{P}<0.05)$. The specific scoring results are shown in Table 3.

\begin{tabular}{|c|c|c|c|c|c|c|}
\hline Group & $\begin{array}{l}\text { The } \\
\text { number } \\
\text { of cases }\end{array}$ & macrosomia & hypoglycemia & $\begin{array}{l}\text { Low blood } \\
\text { calcium }\end{array}$ & hyperbilirubinemia & $\begin{array}{l}\text { Fetal } \\
\text { death } \\
\text { palace }\end{array}$ \\
\hline Traditional & & $8(15.38)$ & $9(17.31)$ & $4(7.69)$ & $7(13.46)$ & 0 \\
\hline $\begin{array}{l}\text { Chinese } \\
\text { Medicine }\end{array}$ & $\mathrm{N}=52$ & & & & & \\
\hline \multicolumn{7}{|l|}{ Group } \\
\hline Western & & $7(13.46)$ & $6(11.54)$ & $2(3.84)$ & $5(9.61)$ & $1(1.92)$ \\
\hline Medicine & $\mathrm{N}=52$ & & & & & \\
\hline \multicolumn{7}{|l|}{ Group } \\
\hline Study Group & $\mathrm{N}=52$ & $2(3.84)$ & $3(5.77)$ & 0 & $1(1.92)$ & 0 \\
\hline$X^{2}$ & & 4.03 & 4.62 & 3.51 & 5.78 & 0.53 \\
\hline $\mathrm{P}$ & & $<0.05$ & $<0.05$ & $<0.05$ & $<0.05$ & $<0.05$ \\
\hline
\end{tabular}

Table 3. Comparison of neonatal outcomes after a course of treatment among pregnant women in three groups [n (\%)]

\section{Conclusion}

Among the drugs for the treatment of gestational cannot pass through the placenta, it is safe for the fetus. Insulin can effectively reduce the blood sugar of pregnant women and improve maternal and infant outcomes. ${ }^{[11]}$ Mendong insulin is used for the treatment of gestational diabetes mellitus, which is easy to inject, has a good effect on the control of postprandial blood glucose, and can also reduce the risk of hypoglycemia before meals. However, the use of Mendong insulin injection alone cannot achieve the best effect, for example, it cannot improve the discomfort symptoms of pregnant women, such as self-induced dry diabetes, insulin is a commonly used drug. Because it mouth and fatigue. ${ }^{[12,13]}$

According to Chinese medicine, gestational diabetes occurs mainly because pregnant women's viscera functions are in a weak state. And after pregnancy, diet is not regular, energy and blood under the accumulation to feed fetus with the continuous growth of the fetus, so the mother's energy and Yin consumption gradually increased, resulting in energy and Yin deficiency of pregnant women. ${ }^{[14]}$ According to the cause of gestational diabetes, radix astragali ophiopogonis decoction can be used to treat puerpera. Studies have shown that astragalus ophiopogonis decoction can inhibit maternal reactive oxygen free radicals, thereby reducing islet cell damage, and promote insulin secretion. ${ }^{[15]}$

Through this study it can be found that after the end 
of a course of treatment, use of astragalus membranaceus dwarf lilyturf soup combined insulin treatment of gestational diabetes pregnant women in blood sugar control and maternal and infant outcomes is superior to the traditional Chinese medicine and western medicine group. The differences were statistically significant, $\mathrm{P}<$ 0.05 , and obviously improves the pregnant woman consciousness of some discomfort symptoms. Modern pharmacology shows that the extracts of astragalus polysaccharide and coumarin have the effect of lowering blood sugar. However, the polysaccharide extracted from ophiopogon japonicus can significantly inhibit the glycemic action of epinephrine. ${ }^{[16]}$

In conclusion, astragalus ophiopogonis decoction combined with Mendong insulin is effective in the treatment of gestational diabetes. Medical personnel should first help pregnant women to establish a healthy lifestyle, and then refer to the scientific use of drugs for pregnant women. ${ }^{[17]}$ This not only helps pregnant women control blood sugar index, but also can effectively improve pregnancy outcome, and improve the quality of life of pregnant women. Therefore, astragalus ophiopogonis decoction combined with Mendong insulin is worthy of application and promotion in the treatment of gestational diabetes.

\section{References}

1. International Association of Diabetes and Pregnancy Study Groups Consensus Panel, Metzger $\mathrm{BE}$, Gabbe $\mathrm{SG}$, et al. International association of diabetes and pregnancy study groups recommendations on the diagnosis and classification of hyperglycemia in pregnancy. Diabetes Care 2010; 33: 676-682. doi: 10.2337/dc10-0544.

2. Maghbooli Z, Hossein-nezhad A, Mirzaei K, et al. Association between retinol-binding protein 4 concentrations and gestational diabetes mellitus and risk of developing metabolic syndrome after pregnancy. Reproductive Sciences 2010; 17(2): 196. doi: $10.1177 / 1933719109351097$

3. Kuzmicki M, Telejko B, Szamatowicz J, et al. High resistin and interleukin- 6 levels are associated with gestational diabetes mellitus. Gynecological Endocrinology 2009; 25(4): 258-263. doi: $10.1080 / 09513590802653825$.

4. $\mathrm{Hu} \mathrm{L}$, Jiang B, Zhang Y. Effects of puerarin combined with insulin aspart on oxidative stress, insulin resistance and pancreatic $\beta$-cell function in pregnant women with gestational diabetes mellitus. Hebei Medical Journal 2016; 38(18): 2761-2764. doi: 10.3969/j.issn.1002-7386.2016.18.011.

5. Hua Y, Jiang $\mathrm{C}, \mathrm{Wu} \mathrm{J}$, et al. Effects of high-dose Vitamin D injection combined with mendong insulin on insulin sensitivity and parathyroid hormone levels in pregnant women with gestational diabetes (in Chinese). China Pharmaceuticals 2014; 23(12): 38-40.

6. Wang $\mathrm{H}, \mathrm{Wu}$ J. Effect of huangqi sijunzi decoction on the treatment of gestational diabetes and its effect on serum c-reactive protein, $\mathrm{Mg} 2+$ and adiponectin levels (in Chinese). Modern Journal of Integrated Traditional Chinese and Western Medicine 2015; 24(26): 2927-2929. doi: 10.3969/j.issn.1008-8849.2015.26.028.

7. Ren Z, Li G. Study on the effect of insulin aspart in treatment of patients with gestational diabetes and its influence on cardiac function in offspring. Journal of Clinical and Experimental Medicine 2015; 14(2): 122-125. doi: 10.3969/j.issn.1671-4695.2015.02.016.

8. Chinese Journal of Diabetes 2017; 25(4): 325-329.

9. Liu M, Qin Y, Xing J. Effect of insulin aspart combined with Huangqi sijunzi decoction on gestational diabetes mellitus and its influence on pregnancy outcomes. Chinese Journal of Woman and Child Health Research 2017; 28(7): 874-876. doi: 10.3969/j.issn.1673-5293.2017.07.040.

10. Xie L, Zhao D, Li S. Effect of metformin combined with Huangqi sijunzi decoction on pregnancy outcome of pregnant women with gestational diabetes (in Chinese). Liaoning Journal of Traditional Chinese Medicine 2016; 43(8): 1678-1680.

11. Cheng H, Mei B. Effects of individualized nutrition intervention combined with astragalus sijunzi decoction on pregnancy outcomes of pregnant women with gestational diabetes (in Chinese). Maternal \& Child Health Care of China 2016; 31(6): 1317-1319.

doi: 10.7620/zgfybj.j.issn.1001-4411.2016.06.77.

12. Li Q, Gao Y, Zhang J. Analysis of the effect of insulin pump on gestational diabetes on pregnancy outcome (in Chinese). Contemporary Medicine 2019; 26(19): 120-122.

13. Zhang B, Chang M. Insulin resistance and islet function changes in pregnant women with gestational diabetes before and after delivery (in Chinese). Clinical Medical Research and Practice 2019; 4(19): 87-88.

14. Su F. Clinical study of astragalus granule combined with insulin pump double wave therapy in the treatment of pregnant women with gestational diabetes (in Chinese). Journal of Practical Diabetology 2019; 15(3): 49-50.

15. Zhou H, Xu Q, Cai P. Effect of high-dose vitamin D on prevention and treatment of gestational diabetes mellitus in obese pregnant women. Chinese Journal of General Practice 2017; 15(11): 1911-1914. doi: 10.16766/j.cnki.issn.1674-4152.2017.11.027. 
16. Wang Y, Liu W, Wang J, et al. Clinical observation of mendong insulin combined with biosynthesis of human insulin in the treatment of gestational diabetes (in Chinese). Medical Pharmaceutical Journal of Chinese Peoples Liberation Army 2016; 28(1):
10.3969/j.issn.2095-140X.2016.01.024

17. Pan B, Ma R. Correlation between serum adipokin-1 and chemerin levels and gestational diabetes (in Chinese). Journal of Southern Medical University 2016; 36(9): 1231-1236. doi: 10.3969/j.issn.1673-4254.2016.09.12. 